\begin{tabular}{|c|l|}
\hline Title & A mathematical model for Cantor coding in the hippocampus \\
\hline Author(s) & Y amaguti, Yutaka; Kuroda, Shigeru; Fukushima, Y asuhiro; Tsukada, Minoru; Tsuda, Ichiro \\
\hline Citation & $\begin{array}{l}\text { Neural Networks, 24(1), 43.53 } \\
\text { https://doi.org/10.1016j.neunet.2010.08.006 }\end{array}$ \\
\hline Issue Date & 2011 \\
\hline Doc URL & http://hdl.handle.net/2115/51450 \\
\hline Type & article (author version) \\
\hline File Information & Neural Networks244353.pdf \\
\hline
\end{tabular}

Instructions for use 


\title{
A Mathematical Model for Cantor Coding in the Hippocampus
}

\author{
Yutaka Yamaguti ${ }^{\mathrm{a}, \mathrm{b}, *}$, Shigeru Kuroda ${ }^{\mathrm{a}}$, Yasuhiro Fukushima ${ }^{\mathrm{c}}$, Minoru \\ Tsukada $^{\mathrm{c}}$, Ichiro Tsuda ${ }^{\mathrm{a}, \mathrm{b}}$ \\ ${ }^{a}$ Research Institute for Electronic Science, Hokkaido University, Kita-20, Nishi-10, Kita-ku, \\ Sapporo, Hokkaido 001-0020, Japan \\ ${ }^{b}$ Research Center for Integrative Mathematics, Hokkaido University, Kita-20, Nishi-10, \\ Kita-ku, Sapporo, Hokkaido 001-0020, Japan \\ ${ }^{c}$ Brain Science Institute, Tamagawa University, 6-1-1 Tamagawa-gakuen, Machida, Tokyo \\ 194-8610, Japan
}

\begin{abstract}
Recent studies suggest that the hippocampus is crucial for memory of sequentially organized information. Cantor coding in hippocampal CA1 is theoretically hypothesized to provide a scheme for encoding temporal sequences of events. Here, in order to investigate this Cantor coding in detail, we construct a CA1 network model consisting of conductance-based model neurons. It is assumed that CA3 outputs temporal sequences of spatial patterns to CA1. We examine the dependence of output patterns of CA1 neurons on input time series by taking each output and combining it with an input sequence. It is shown that the output patterns of CA1 were hierarchically clustered in a self-similar manner according to the similarity of input temporal sequences. The population dynamics of the network can be well approximated by a set of contractive affine transformations, which forms a Cantor set. Furthermore, it is shown that the performance of the encoding scheme sensitively depends on the interval of input sequences. The bursting neurons with NMDA synapses are effective for encoding sequential input with long (over $150 \mathrm{msec}$ ) intervals while the non-bursting neurons with AMPA synapses are effective for encoding input with short (less than $30 \mathrm{msec}$ ) intervals.
\end{abstract}

Keywords: hippocampus, Cantor coding, iterated function system

\section{Introduction}

One of the crucial factors in processing episodic memory is the representation of a temporal sequence of events. According to Tulving [44], episodic memory is the memory of individual experiences. In humans, the hippocampus is a

\footnotetext{
*Corresponding author. Tel.: +81-11-706-9449; Fax: +81-11-706-9450.

Email address: yamaguchi@math.sci.hokudai.ac.jp (Yutaka Yamaguti)
}

Preprint submitted to Neural Networks 
necessary organ for the formation of episodic memory [28, 33], and recent studies in rodents also suggest the involvement of hippocampal cells in the processing of context-dependent and/or sequentially represented episodic-like memories [5, 47].

The hippocampal CA3 region has dense recurrent connections between pyramidal neurons. Since the seminal work of Marr [18], CA3 has been considered to be involved in associative memory [19, 35, 14], and recent experimental evidence has appeared in support of this notion [46, 21]. As for the function of the CA1 region, some researchers have indicated that it is tuned for the processing of temporal information [15, 16, 43]. Recently, Yoshida \& Hayashi [49] constructed a model of the learning of sequential input from CA3 to CA1.

Here, we investigate the information representation in CA1 neurons when the CA1 network receives time-dependent activity output mainly from CA3. Various kinds of rhythmic activity have been observed in the hippocampus and neighboring areas $[2,4,45]$, especially theta and gamma oscillations during the encoding and retrieval of memory [29, 30, 20]. The continuous input of stimuli may synchronize with these rhythms [17]. If this were the case, it would be reasonable to assume that the input stimuli are internally transformed into a discrete time series of activity. Given these considerations, in this paper, we assume that the input time series from CA3 to CA1 is discrete but not continuous. In other words, we assume that the input time series consists of several elementary spatial patterns of neuronal activity. Whether CA3 produces temporal activity in associative memory is still unknown. However, the model studies by Tsuda and colleagues $[38,36]$ have indicated the possibility of the presence of dynamic associative memory in CA3.

"Cantor coding" in CA1 was first proposed by Tsuda and colleagues [37, 39]. In their hypothesis, Cantor coding provides the scheme for encoding temporal sequences of events and it forms a fractal-like hierarchical structure in the state space of neural dynamics. Tsuda and Kuroda proposed a simple CA3CA1 model in which CA1 encodes the sequence. Subsequent observations by Fukushima, Tsukada, Tsuda, Yamaguti, \& Kuroda [9] of hierarchical representations of input time series in the membrane potential of CA1 neurons have suggested the presence of Cantor coding in the actual hippocampus.

Therefore, here we aim to develop a biology-oriented model of CA1 in order to study the possibility of Cantor coding in CA1. Then, we investigate the physiologically plausible condition in which Cantor coding could emerge and provide some predictions on the possibility of observation of Cantor coding in experimental studies.

The rest of the paper is organized as follows. In Section 2, the basic notion of Cantor coding is explained. In Section 3, our computational model of CA1 is described. In Section 4, we show our computational results. The coding performance is estimated and its dependence of the coding performance on the interval is treated in Section 5. Section 6 provides a summary and discussion of the work. 


\section{Cantor coding}

We use the term 'Cantor coding' to mean the encoding of time series information by a Cantor set [31]. Here, a Cantor set is a kind of fractal set, which is defined as a non-empty set which is (i) closed, (ii) perfect, and (iii) totally disconnected [see, for example, 25]. This set has a self-similar structure. In early studies of recurrent neural networks $[6,24,34]$, it was reported that Cantor sets emerge in the internal state space of neural networks, which deal with temporal sequences such as language and motor commands. Tsuda and colleagues have proposed a Cantor coding scheme in chaos-driven contracting systems [40], and discussed the possibility of Cantor coding for the formation of episodic memory in the hippocampus [37, 39]. Using a simple hippocampal model, they showed that transitions between so-called quasi-attractor states occur in the CA3 recurrent network, and that the path of the state transitions can be hierarchically encoded on the Cantor set in CA1. To address the biological plausibility of this process, here we construct a biologically oriented model which represents the physiological neural networks of CA1. We will show that each element of the Cantor set represents a specific time series.

A Cantor set can be generated by a dynamical system. One method to create a Cantor set is to use chaos-driven contracting maps, where one can observe a Cantor set in a subspace, which is taken not to see the chaotic component $[26,40,27]$.

Another method to create a Cantor set is to use an iterated function system (IFS) $[10,1,7]$. IFS is defined by a compact metric space $M$ and a set of maps $\mathcal{F}=\left\{f_{1}, \ldots, f_{m}\right\}$, where $f_{i}: M \rightarrow M$ and $m \geq 2$. If all of maps are 'contractive', a unique attractor $A \subset M$ which satisfies $A=\bigcup_{i=1}^{m} f_{i}(A)$ exists. The attractor is usually a fractal. Here 'contractive' means that the distance between any two points is shortened by applying the map under a given metric. Furthermore, the attractor $A$ is totally disconnected and forms a Cantor set if the non-overlapping condition $f_{i}(A) \cap f_{j}(A)=\emptyset$ for all $i \neq j$ is satisfied.

Now, let us consider a set of transformations $\mathcal{F}=\left\{f_{1}, \ldots, f_{m}\right\}$. Take $x_{0} \in M$ as an initial point, select one contractive transformation $f_{i_{0}}$ from $\mathcal{F}$ at random, and let $x_{1}=f_{i_{0}}\left(x_{0}\right)$. Repeat this procedure to obtain a sequence of points $\left\{x_{t}\right\}$. For large enough $t$, the distance between points $x_{t}$ and $A$ will converge to zero, with $x_{t}$ close to $f_{i_{t}} \circ \cdots \circ f_{i_{1}}(A)$. Here, the distance between a point $x$ and a set $A$ is given by $d(x, A)=\inf _{a \in A} d(x, a)$, where $d(x, a)$ is a distance function underlying the metric space $M$. The sequence $\left\{x_{t}\right\}$ will randomly distribute across $A$ so that the plot of the long sequence $\left\{x_{t}\right\}$ gives a good approximation of the attractor $A$ in the sense of measure theory. In the case that $A$ is totally disconnected, there are one-to-one correspondences between the space of an infinite sequence of symbols $\omega=j_{1}, j_{2}, \ldots,\left(j_{k} \in\{1, \ldots, m\}\right)$ and $A$ by mapping $\omega$ to a limit of $f_{j_{1}} \circ f_{j_{2}} \circ \cdots(A)$. In this way, every point on the Cantor set corresponds to one sequence of the symbols, and the distance between two symbol sequences is naturally mapped to the distance between two points on the Cantor set. 


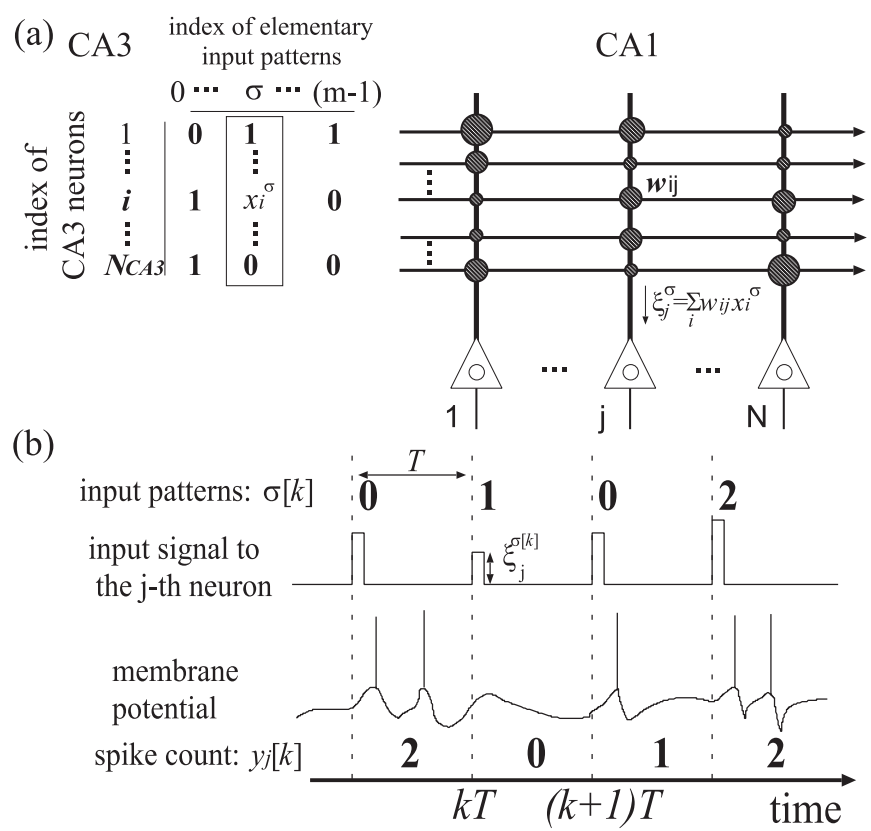

Fig. 1: Schematic of the CA1 network.(a) Inputs from CA3, and the CA1 network receiving such inputs. (b) Representation of input time series to the $j$-th CA1 neuron and response of the CA1 neuron.

\section{Biology-oriented Model}

\subsection{Constitution of the CA1 network with CA3 outputs}

In this section, we propose a model of the CA1 network for Cantor coding. Fig. 1 shows a schematic drawing of our CA1 network receiving sequential inputs from CA3. As a first step, in order to clarify the role of excitatory pyramidal neurons, here we neglect interneurons.

The CA3 network is assumed to store $M$ spatial firing patterns $\left\{X^{0}, X^{1}, \ldots, X^{M-1}\right\}$, which we call elementary patterns. One may conceive that each elementary pattern corresponds to an elementary event of sequentially organized episodic memory. Each elementary pattern can be embedded in the CA3 network by hetero- or auto-associative connections. Each pattern $X^{\sigma}=\left(x_{1}^{\sigma}, \ldots, x_{N_{\mathrm{CA} 3}}^{\sigma}\right)$, $(\sigma \in\{0, \ldots, M-1\})$ consists of $N_{\mathrm{CA} 3}$ elements. Each element $x_{i}^{\sigma}$, which represents the state of the $i$-th CA3 neuron, is assigned a value of either 1 or 0 randomly and independently. The probability of taking value 1 is $p_{f i}=0.1$ throughout this paper.

The model CA1 consists of $N$ pyramidal neurons. These neurons receive a temporal series of elementary patterns via AMPA- and NMDA-receptor mediated synaptic activity. The synaptic strength $w_{i j}$ from the $i$-th neuron of CA3 
to the $j$-th neuron of $\mathrm{CA} 1$ is given by the following Hebbian-type rule:

$$
w_{i j}=\tilde{w} \sum_{\sigma=0}^{M-1} x_{i}^{\sigma} y_{j}^{\sigma}
$$

where $y_{j}^{\sigma}$ is the activity of the $j$-th neuron for the response to input pattern $\sigma$, and $\tilde{w}$ is an adjustable parameter for the control of the input strength. Each $y_{j}^{\sigma}$ is a real number randomly chosen from uniform distribution in $[0,1]$. Later, we will also show another type of distribution of $y_{j}$ (See, Sec. 5.3). For simplicity, the same weight $w_{i j}$ is used for both AMPA and NMDA synapses. The sum of the synaptic inputs of the $j$-th neuron for the input pattern $X^{\sigma}$ is represented by the following equation.

$$
\xi_{j}^{\sigma}=\sum_{i=1}^{N_{\mathrm{CA} 3}} w_{i j} x_{i}^{\sigma} .
$$

As already mentioned, we consider the situation that a rhythmically switching sequential pattern is delivered to the CA1 network. One of the $m(<M)$ elementary input patterns defined above is randomly chosen every $T$-msec. The probability to select any pattern is equally given, that is, $(1 / m)$. We denote the index of the input pattern selected at time $t=k T$ by $\sigma[k] \quad(k=0,1,2, \ldots, L)$. The duration of the input stimuli takes $\Delta=5 \mathrm{msec}$. To summarize, the input signal to the $j$-th neuron in CA1 is given by

$$
s_{j}(t)= \begin{cases}\xi_{j}^{\sigma[k]} & k T<t<k T+\Delta, \quad k=0,1,2, \ldots \\ 0 & \text { otherwise. }\end{cases}
$$

\subsection{A single neuron model}

We use the two-compartment model proposed by Pinsky \& Rinzel [23] as the model of a pyramidal neuron in the hippocampus. This model can describe the electric properties of both dendrite and cell body, each of which is assumed to be electrically uniform. The dynamics of the model neuron are given as follows:

$$
\begin{aligned}
C_{m} d V_{s} / d t= & -I_{L}\left(V_{s}\right)-I_{\mathrm{Na}}\left(V_{s}, m, h\right)-I_{\mathrm{K}-\mathrm{DR}}\left(V_{s}, n\right) \\
& +\left(g_{c} / p\right)\left(V_{d}-V_{s}\right)+I_{s} / p \\
C_{m} d V_{d} / d t= & -I_{L}\left(V_{d}\right)-I_{\mathrm{Ca}}\left(V_{d}, s\right)-I_{\mathrm{K}-\mathrm{AHP}}\left(V_{d}, q\right) \\
& -I_{\mathrm{K}-\mathrm{C}}\left(V_{d},\left[\mathrm{Ca}^{2+}\right], c\right)-\left(I_{\mathrm{AMPA}}+I_{\mathrm{NMDA}}\right) /(1-p) \\
& +\left(g_{c} /(1-p)\right)\left(V_{s}-V_{d}\right)+I_{d} /(1-p) .
\end{aligned}
$$

Here, variables $V_{s}$ and $V_{d}$ are membrane potentials of the cell body and dendrite, respectively. $C_{m}$ is the conductance of the membrane, $g_{c}$ is the conductance between the soma and dendritic compartments, and $p$ is the ratio of the area of soma to dendrite. $I_{\mathrm{Na}}, I_{\mathrm{K}-\mathrm{DR}}, I_{\mathrm{Ca}}, I_{\mathrm{K}-\mathrm{AHP}}$, and $I_{\mathrm{K}-\mathrm{C}}$ are ionic currents associated with different types of ionic channels, $I_{\mathrm{L}}$ is the leak current, $I_{\mathrm{AMPA}}$ and $I_{\mathrm{NMDA}}$ 
are synaptic currents, and $I_{s}\left(I_{d}\right)$ is the current externally applied to the soma (dendrite).

The gating variables for representing the fraction of open ion channels for $I_{\mathrm{Na}}, I_{\mathrm{K}-\mathrm{DR}}, I_{\mathrm{Ca}}, I_{\mathrm{K}-\mathrm{AHP}}$, and $I_{\mathrm{K}-\mathrm{C}}$ are denoted by $m, h, n, s, c$, and $q$, respectively. These kinetic equations take the form

$$
d y / d t=\alpha_{y}(1-y)-\beta_{y} y,
$$

where the variable $y$ stands for $h, n, s, c$, and $q$. Both $\alpha_{y}$ and $\beta_{y}$ are either voltage-dependent $(h, n, s, c)$ or $\left[\mathrm{Ca}^{2+}\right]$-dependent $(q)$ functions. The gating variable $m$ is substituted by its steady-state function

$$
m_{\infty}\left(V_{s}\right)=\alpha_{m}\left(V_{s}\right) /\left(\alpha_{m}\left(V_{s}\right)+\beta_{m}\left(V_{s}\right)\right) .
$$

The full equations of ionic currents $I_{x}, \alpha_{y}$ and $\beta_{y}$ are described in Appendix A.

The variable $\left[\mathrm{Ca}^{2+}\right]$ is a unitless quantity and represents the intra-cellular free calcium level in the dendritic compartment. Its dynamics is described by

$$
d\left[\mathrm{Ca}^{2+}\right] / d t=-a_{\mathrm{Ca}} I_{\mathrm{Ca}}-b_{\mathrm{Ca}}\left[\mathrm{Ca}^{2+}\right] .
$$

\subsection{Synaptic connections}

Two types of synaptic conductance are used in the present model: conductance via an AMPA receptor and that via an NMDA receptor. AMPA conductance is fast rising and fast decaying while NMDA conductance is fast rising but slow decaying. Both receptors are located in the dendritic compartment. The AMPA receptor-mediated synaptic current $I_{\mathrm{AMPA}}$ is given by

$$
I_{\mathrm{AMPA}}(t)=\tilde{g}_{\mathrm{AMPA}} G_{a}\left(V_{d}-V_{\mathrm{AMPA}}\right),
$$

where $\tilde{g}_{\mathrm{AMPA}}$ is the parameter measuring absolute AMPA conductance and $V_{\mathrm{AMPA}}$ is the reversal potential. $G_{a}$ is the gating variable activated by the presynaptic inputs, which is described by

$$
d G_{a} / d t=s_{a}-G_{a} / 2
$$

where $s_{a}(t)$ represents a presynaptic input from CA3, which is described in Eq. (3).

The NMDA receptor-mediated synaptic current $I_{\mathrm{NMDA}}$ is given by

$$
I_{\mathrm{NMDA}}(t)=\tilde{g}_{\mathrm{NMDA}} G_{n}\left(1+0.28 \exp \left(-0.062\left(V_{d}\right)\right)\right)^{-1}\left(V_{d}-V_{\mathrm{NMDA}}\right),
$$

where $\tilde{g}_{\text {NMDA }}$ is the parameter measuring absolute NMDA conductance and $V_{\mathrm{NMDA}}$ is the reversal potential. Similarly to the AMPA synapse, $G_{n}$ is the gating variable and its dynamics is given by

$$
d G_{n} / d t=s_{n}(t)-G_{n} / 150,
$$

where $s_{n}(t)$ represents a presynaptic input.

The full description of the model and values of the parameters are described in Appendix A. 

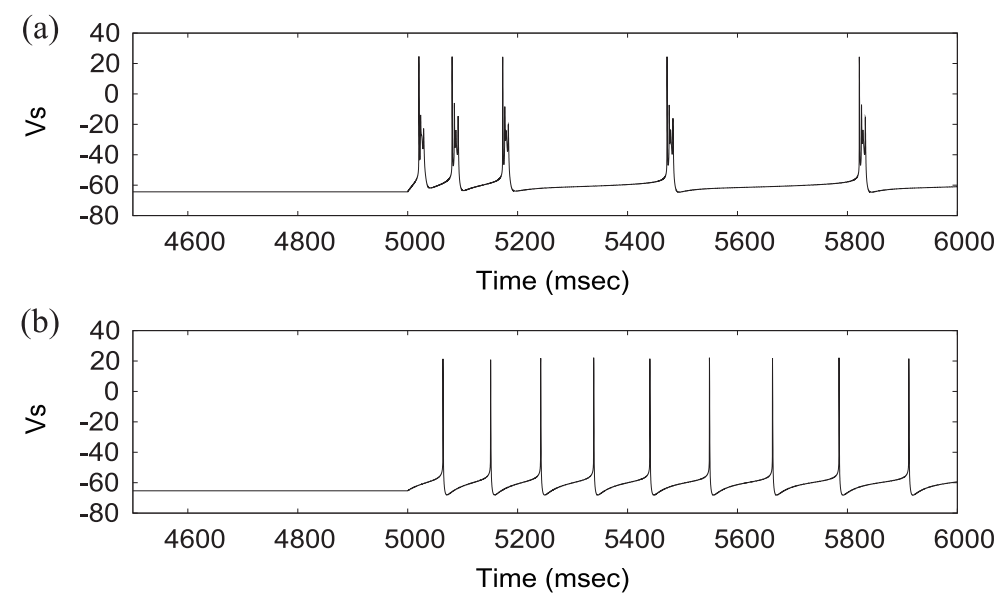

Fig. 2: Firing patterns produced by (a) bursting type and (b) spiking type (b) neurons.

\subsection{Parameters}

We use two parameter sets for ion channels which determine the electric properties of neurons. In the first set, the parameter values used in the paper by Pinsky \& Rinzel [23] are employed. With these parameter values, the neuron tends to show bursting behavior in response to a constant current (Fig. 2(a)). The parameter values of the second set are modified in order to imitate the activity of a regular spiking cell $[3,11]$, in which spontaneous bursting activity rarely occurs (Fig. 2(b)). We denote these parameter sets as bursting type and spiking type, respectively. Both types of pyramidal neurons have been found in rat hippocampal CA1 slices [13, 12].

Furthermore, we use two parameter sets for synaptic strength $g_{\mathrm{AMPA}}$ and $g_{\mathrm{NMDA}}$, in order to study the effect of these two synaptic types on coding performance. In the first set denoted by the AMPA type, only AMPA synapses are available $\left(g_{\mathrm{AMPA}}=0.01\right.$ and $\left.g_{\mathrm{NMDA}}=0\right)$. In the other set denoted by the NMDA type, both the AMPA and NMDA types of synapses are used for synaptic transmission $\left(g_{\mathrm{AMPA}}=0.004\right.$ and $\left.g_{\mathrm{NMDA}}=0.01\right)$. In Section 4 , we use bursting type neurons with AMPA and NMDA synapses.

\subsection{Output of the network}

We use two quantities representing internal state and outputs of the CA1 network. One is the average of the somatic membrane potential during the interval between two successive inputs of elementary patterns. We denote a vector consisting of the averaged values by

$$
\bar{v}[k]=\left(\bar{v}_{1}[k], \bar{v}_{2}[k], \ldots, \bar{v}_{N}[k]\right)^{T} .
$$

Here, $\bar{v}_{j}[k]=(1 / T) \int_{k T}^{(k+1) T} V_{s, j}(t) d t$, where $V_{s, j}(t)$ represents the somatic membrane potential of the $j$-th neuron. 
The other quantity represents the vector consisting of the numbers of spikes yielded in the intervals between two successive inputs of elementary patterns:

$$
y[k]=\left(y_{1}[k], y_{2}[k], \ldots, y_{N}[k]\right)^{T},
$$

where $y_{j}[k]$ is the number of spikes of the $j$-th neuron in the interval $(k T,(k+$ 1)T). Here, depolarizations of the somatic membrane potential above the threshold $V_{t h}=0$ are considered as spikes.

It should be noted that the output pattern $y[k]$ does not depend solely on the most recent elementary input pattern $\sigma[k]$ but also depends on the internal state of the network at time $t=k T$, which in turn depends on inputs from the more distant past. In this sense, the output vector reflects the history of the input sequence. The manner of its dependency is, however, not trivial. In Section 4, we will show that the output vector can encode the input sequence in a self-similar manner.

Let us introduce some notations for sequential input patterns. Let $\sigma[k]$ be the index of the input patterns at $t=k T$, and

$$
\sigma^{d}[k]=(\sigma[k], \sigma[k-1], \ldots \sigma[k-d+1])
$$

be the input sequence of length $d$ from the $(k-d+1)$-th step to the $k$-th step. We investigate the relation between the input sequences $\sigma^{d}[k]$ with the output vectors $\{\bar{v}[k]\}$ and $\{y[k]\}$, and analyze distributions of the output vectors conditioned by the input sequences. Fig. 1-(b) illustrates a possible response of the neuron to an input sequence.

\subsection{Numerical simulations}

For each numerical simulation, the differential equations were numerically integrated from randomly chosen initial conditions and 10,000 output vectors $\{y[k]\}$ with input sequences were recorded after discarding transient data during 100T. The 4th-order Runge-Kutta method with a time step of $0.05 \mathrm{msec}$ was used to integrate the equations. All simulations were performed on Intel Xeon processor machines with Linux and FreeBSD operating systems. Programs for the computation were written in $\mathrm{C}++$ language. To examine the validity of the numerical results, we repeated similar simulations with smaller $(0.005 \mathrm{msec})$ and larger $(0.1 \mathrm{msec})$ time steps and found no significant differences in the results.

\section{Computational Results}

\subsection{Hierarchical structure}

First, we show a typical example of self-similar structure emerging in internal state space and output vector space. In order to understand how the distributions of $\bar{v}[k]$ and $y[k]$ are formed in the high-dimensional spaces ( $N$-dimension), we utilize principal component analysis (PCA), transforming high-dimensional vectors to a lower-dimensional space(say, $l$-dimension). PCA allows us to reduce the $N$-dimension space to the $l$-dimension space by finding the optimal 
transformation, thereby minimizing the sum of squared distances between the original and the transformed points.

The transformation is given by

$$
u[k]=V^{T} \bar{v}[k],
$$

where $V$ is a $N \times l$ matrix consisting of eigenvectors of $l$ larger eigenvalues of the scatter matrix

$$
C=\frac{1}{L} \sum_{k=0}^{L-1}(\bar{v}[k]-\bar{m})(\bar{v}[k]-\bar{m})^{T},
$$

where $L$ is the total number of obtained data and $\bar{m}$ is the mean of $\bar{v}$.

The first example is shown in Fig. 3, where average membrane potentials $\bar{v}[k]$ with weak inputs are plotted, which triggers no spike of the CA1 neurons $(m=3$, $T=150$, and $\tilde{w}=0.02$ ). The distribution obtained looks like a self-similar set. Each color represents a different input sequence at each length of sequence $d$ $(=1,2$ and 3 from left to right in Fig. 3). The distribution shown in Fig. 3(a) is roughly split into three sub-clusters, each of which corresponds to the elementary pattern of input, denoted by '0', '1', and '2'. Furthermore, each sub-cluster can be divided into three more sub-clusters; for example, cluster corresponding to the input sequence ' 0 ' is composed of three sub-clusters corresponding to input sequences '00','01' ,'02', as shown in Fig. 3(b). This hierarchy of clusters is further observed for a longer length input sequence, as shown in Fig. 3(c).

Interestingly, a similar result was obtained in the space of spiking output patterns $\{y[k]\}$ under relatively strong input from CA3. Fig. 4 shows a typical example for the plots of output patterns $\{y[k]\}$ under the super-threshold condition of $m=3, T=100$, and $\tilde{w}=1.6$. In this way, hierarchical structure emerged in the distribution of the output patterns. This suggests the existence of Cantor sets in actual hippocampal neurons.

In both cases, similarity between the input sequences is represented by, for example, a Hausdorff distance. Hausdorff distance is a metric that measures differences of two subsets of a metric space $(M, d)$, where $d$ is a metric. Let a $\delta$-neighborhood of a subset $A$ be $A_{\delta}=\{x \in M \mid d(x, a) \leq \delta$ for some $a \in A\}$. The Hausdorff distance between two non-empty subsets $A$ and $B$ is defined as

$$
d_{H}(A, B)=\inf \left\{\delta \mid A \subset B_{\delta} \text { and } B \subset A_{\delta}\right\} .
$$

Here, we define subsets of the vectors of averaged membrane potentials $\bar{v}[k]$ indexed by an input sequence $\sigma^{d}=\left(\sigma_{1}, \ldots, \sigma_{d}\right)$ as $V\left(\sigma^{d}\right)=\left\{\bar{v}[k] \mid \sigma^{d}[k]=\right.$ $\sigma^{d}$ for $\left.k=1,2, \ldots\right\}$. Let us define the similarity between the two different input sequences of length $d, \sigma^{d}$ and $\sigma^{\prime d}$ as

$$
\operatorname{Sim}\left(\sigma^{d}, \sigma^{\prime}\right)=\min \left\{i \mid \sigma_{i} \neq \sigma_{i}^{\prime}\right\}-1
$$

We calculated Hausdorff distance between all possible pairs of $V\left(\sigma^{d}\right)$ and $V\left(\sigma^{\prime} d\right)$ for $d=5$, and then, calculated an average for each similarity. The distance clearly decreases with the increases of similarity (Fig. 5, dashed line). That tendency is more sharply observed if we reduce the dimension by PCA and calculate the Hausdorff distance on the reduced space (Fig. 5, solid line). 
(a)

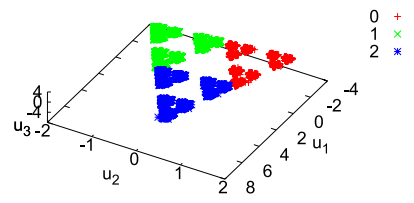

(b)

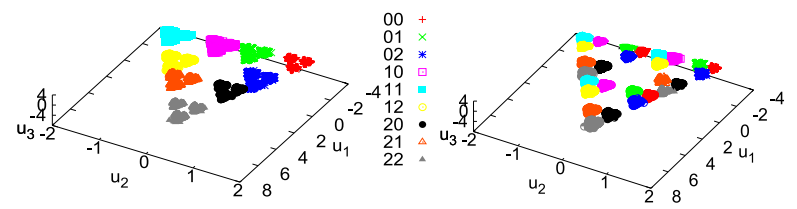

Fig. 3: Distribution of $\{\bar{v}[k]\}$. Here, $m=3 \tilde{w}=0.02$, and $T=150$. Colors indicate input sequences $\sigma^{d}[k]$ that CA1 received just before the outputs. Length of sequence $d$ is 1 (a), 2 (b), and 3 (c).

(a)

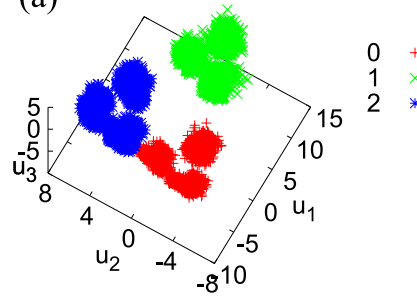

(b)

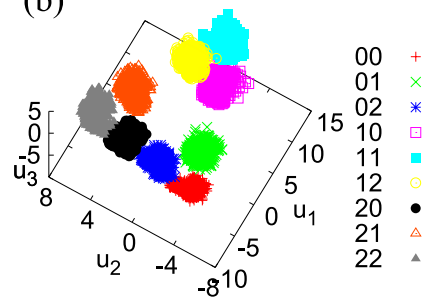

(c)

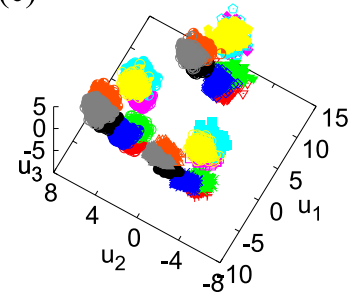

Fig. 4: Distribution of output vectors $\{y[k]\}$. Here, $m=3 \tilde{w}=1.6$, and $T=100$. Length of sequence $d$ is $1(\mathrm{a}), 2(\mathrm{~b})$, and 3 (c).

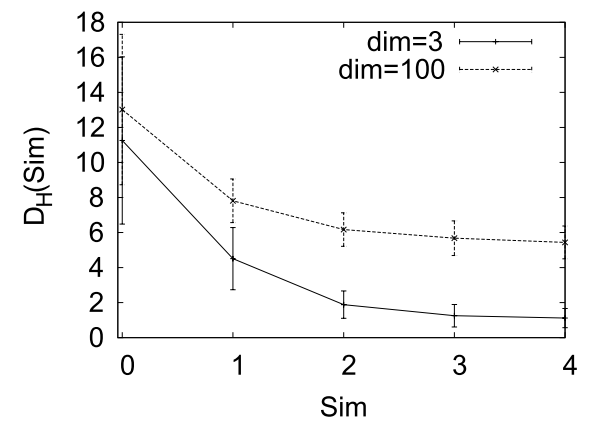

Fig. 5: Mean Hausdorff distances as a function of similarity between two input sequences. 

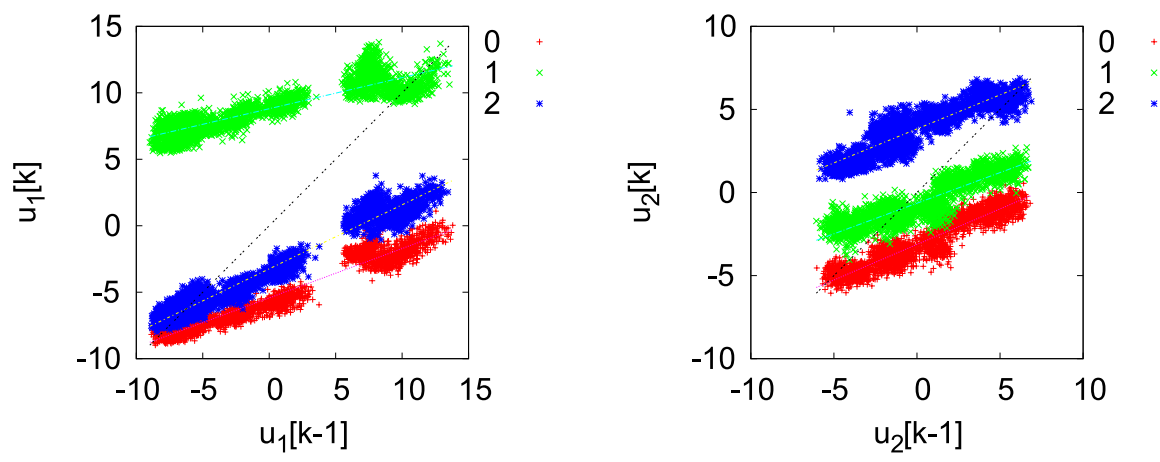

Fig. 6: Return maps of first (left) and second (right) principal components of CA1 output patterns. Each color indicates a kind of elementary input pattern $\sigma[k]$. Parameter values are the same as in Fig. 4.

\subsection{Return maps}

How can these Cantor sets be yielded? One possible mechanism generating such sets is the use of an IFS (see Section 2). To clarify if a contractive IFS emerges in the CA1 dynamics, we investigated the return maps for the principal components $u_{i}(i=1$ and 2$)$ of the output patterns $y[k]$. Here, the return map for each $i$ is obtained by plotting the values at time $k, u_{i}[k](k=1,2, \ldots$, versus $u_{i}[k-1]$ at time $k-1$. The return maps showed that the CA1 dynamics up to the second components are well approximated by one-dimensional affine transformations

$$
u_{i}[k]=a_{i}^{\sigma[k]} u_{i}[k-1]+b_{i}^{\sigma[k]},
$$

where $a_{i}^{j}, b_{i}^{j}(i=1,2, j=0, \ldots, m-1)$ are constants, $i$ denotes the component, and $j=\sigma[k]$ is the index of an elementary pattern input at time $k T$ (Fig. 6). Absolute values of slopes $\left|a_{i}^{j}\right|<1$ for all $j$ means that these maps are contractive, which assures the existence of a unique attractor for the present IFS [10]. This result suggests that the CA1 system driven by a sequential input can be viewed as an IFS.

In order to study how these affine transformations arise in the model networks, we observed the return maps of averaged membrane potentials of individual neurons. Fig. 7-(a) shows return maps of principal components of averaged membrane potentials. In this example, the first and the second components can explain most $(>82 \%)$ of the variations in the original space of membrane potentials. We chose four neurons which most contribute to the first two components as an example of the dynamics of individual neurons that contribute to the emergence of Cantor coding. We chose indexes $i_{1}, \ldots, i_{4}$ which give the maximal values of $\left|V_{1, i_{n}}\right|+\left|V_{2, i_{n}}\right|$, where $V_{i, j}$ is $(i, j)$-th entry of the matrix $V$ defined in Eq. (15). Individual return maps of such neurons are shown in Fig. 7-(b). Dynamics of the membrane potential exhibit highly complex dynamics and can not be represented by one-dimensional maps although the mean responses are significantly different depending on inputs. Taking weighted sums 
of these responses, we obtained a clear one-dimensional maps as is show in Fig. 7-(a).

The present model CA1 has the neural network such that the input layer is independently connected to each output neuron via Hebbian learning algorithm. Thus, the low-dimensional behaviors observed in the first and the second principal components cannot stem from a conventional self-organization ability of the interacting neurons. In other words, such low-dimensional behaviors cannot be viewed as so called collective behaviors. Fig. 7-(b) shows the return maps of the most contributing neurons to the low-dimensional behaviors of the first and the second principal components shown in Fig. 7-(a). The return maps of some other neurons are highly random. The return maps of the residual neurons look low-dimensional but are not separable, and thus the different input pattern sequences cannot be distinguished in such neurons, which behave almost periodically. Therefore, these neurons do not contribute to the separation of input patterns. The most contributing neurons are also random, but possess the low-dimensional components of the behaviors, which may stem from the synaptic structure organized by the Hebbian learning. In other words, the correlation between input sequences and outputs can be represented by some neurons. Averaging such correlated neurons, random components are reduced and only deterministic ones remain.

We also investigated the interacting neurons via inhibitory interneurons. In such a case, even the third principal component shows low-dimensional mapping as well as the first and the second components. This may indicate that in addition to the formation of synaptic structure via Hebbian learning, collective behaviors can also be organized in the network as to be able to separate the input pattern sequences. A detailed analysis of the model CA1 with inhibitory neurons will be published elsewhere.

\section{Estimation of Coding Performance}

In order to estimate the performance of the Cantor coding scheme and its dependence on parameters, we quantified the degree of separability among different sets of spike patterns, each of which corresponded to a different input sequence. The procedure is summarized as follows. We take $\left(\sigma^{d}[k], y[k]\right)$ as the input-output pair, where $\sigma^{d}[k]$ is the input sequence from the $(k-d+1)$-th to the $k$-th step and $y[k]$ is the output spiking pattern defined in Eq. (13). First, we conducted a numerical simulation. Second, we divided the time series of the input-output pair into a training data set and a test data set. Third, using the training data set, we constructed a pattern classifier, by which the corresponding input sequences could be inferred from the output spiking patterns. Fourth, the test data set was input to the classifier and the output of the classifier was compared to the true input sequences. Fifth, the error rates of the classification were calculated. By repeating steps 2 through 5 with different training and test data sets, the mean of the error rate (MER) was calculated, which was taken as the measure of separability of the different sets of output spiking patterns which encode the different input sequences. 
(a)
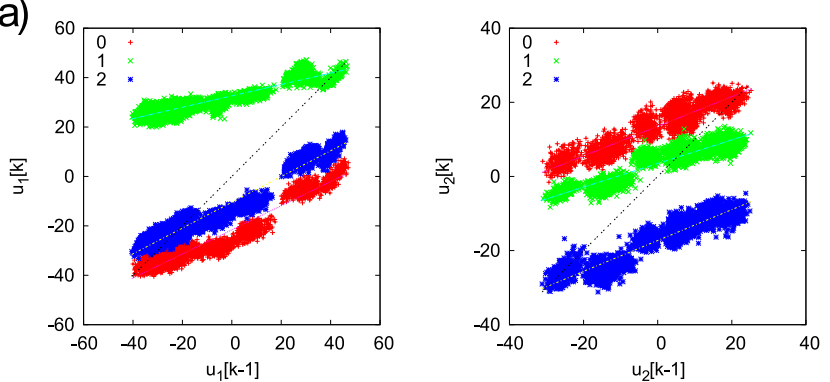

(b)
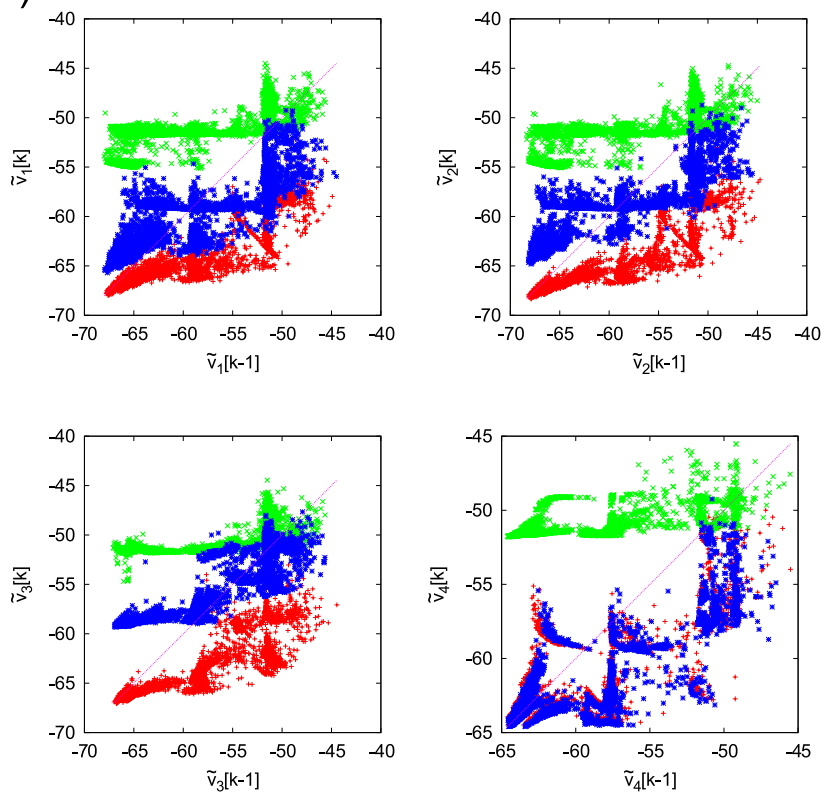

Fig. 7: (a) Return maps of the first (left) and the second (right) principal components of averaged membrane potentials. Data are obtained from the same simulation as Fig. 4. (b) Return maps of averaged membrane potentials of four different neurons. 


\subsection{Linear discriminant analysis}

We utilized linear discriminant analysis (LDA) for the classifier [8]. LDA is a statistical method to classify data represented by vectors into mutually exclusive classes. Here, the input data to classify is a set of output spiking patterns of the CA1 network. The purpose of the classification is to determine the class of each input vector among possible $q$ classes $\left\{\omega_{i}^{d}\right\}(i=1, \ldots, q)$, where $\omega_{i}^{d}$ represents an input sequence of length $d, q=m^{d}$ and $m$ is the number of a kind of input pattern. In LDA, the input vector $y$ is transformed into a low-dimensional space, which is here called $z$-space, by the transformation

$$
z=A^{T} y,
$$

where $A$ is an $N \times(q-1)$ matrix. Using the training data set, the matrix $A$ is determined to maximize the average separation of the projections of points belonging to different classes whilst minimizing the within-class variances of the projections of those points (see Appendix B). Then, the vector $y$ from the test data set is classified to the class $\omega_{i}^{d}$ whose centroid is closest to the point $z=A^{T} y$ in the $z$-space. The details of the algorithm we used here are described in Appendix B. It should be noted that this procedure can be realized by a simple perceptron with appropriate connection weight. It is thus reasonable to expect that neural networks in the neocortex which receive hippocampal outputs can perform a similar processing procedure.

\subsubsection{Procedure}

Here, we mainly study the case $m=2$. We have confirmed that the cases of $m=3$ and 4 show similar results. First, we collect the output patterns for which only the oldest input patterns are different, each corresponding sequence of which belongs to either of two sequences, for example, $\left(\sigma_{i}^{3}==^{\prime} 010^{\prime}, \sigma_{j}^{3}=^{\prime} 011^{\prime}\right)$ or $\left(\sigma_{i}^{4}=^{\prime} 1100^{\prime}, \sigma_{j}^{3}=^{\prime} 1101^{\prime}\right)$. What should be classified are $m^{d-1}$ groups of vectors for each length of sequence $d$. For each group, the task of the LDA classifier is to distinguish output patterns into two classes, each of which corresponds to a particular input sequence. In order to estimate the MER, here we used the cross-validation method. The data set was divided into ten segments. Then, for each segment, using the other nine segments as training sets, we built the matrix $A$ in Eq. (20) and performed the classification. The ratio of the incorrect classification was then calculated. The mean error ratio of the $m^{d-1}$ groups is referred as the MER of the length $d$.

\subsection{A representative case of $L D A$ classification}

Let us examine a typical case of LDA classification. Histograms of $z$ values on $z$-spaces of two similar sequences which should be distinguished are shown in Fig. 8. Since $m=2$, the $z$-space is one dimensional. On the space, the separation of two classes is fairly clear up to $d=5$. Fig. 8-(a) indicates the clear separation of the largest hierarchy of patterns, where the distribution of each class shows a Gaussian-like distribution, so there seems to be no more hierarchies of patterns. However, as shown in Fig. 8-(b), the distribution of $z$ values projecting in a 
different direction shows further separation of the Gaussian-like distributions. This relation is clearly seen up to $d=5$ in the present case.

To investigate the characteristics of the population coding, we studied the dependence of the MER on the number of CA1 neurons (Fig. 9). The MER were exponentially decreased with the number of neurons. This gradual improvement of the coding represents the effectiveness of population coding.

\subsection{Dependence of coding on time interval of successive elementary patterns of input}

In the hippocampus, various rhythmic activities such as $\gamma$ and $\theta$ oscillations have been observed $[2,4]$. To investigate the efficiency of Cantor coding depending on the input time interval, we calculated the MER as a function of the time interval and the strength of input. MERs in the case of interval $T=10,20,30,50,100,150,200,250$ and 300 were calculated for each input strength. We investigated the performance of networks with four different types of parameter sets for ion channels and synaptic property, namely, bursting neurons with active NMDA channels (Bursting-NMDA), spiking neurons with active NMDA channels (Spiking-NMDA), bursting neurons with inactive NMDA channels (Bursting-AMPA), and spiking neurons with inactive NMDA channels (Spiking-AMPA). Here, active NMDA channels means that the NMDA channels are opened and calcium ion influx occurs but this does not serve to increase the synaptic strength, and thus we do not consider any synaptic learning. AMPA channels are assumed to be activated in all cases.

Fig. 10 shows the MERs in the case of $d=3$ as a function of the interval $T$ and the input strength $\tilde{w}$. In the case of the Bursting-NMDA neurons, coding efficacy is relatively good when the interval $T$ is over $150 \mathrm{msec}$. The efficacy was not high, however, in cases where $T<50$. It seems that this comes from the time constants of decay of the NMDA current and slow ion channels such as $I_{\mathrm{K}-\mathrm{AHP}}$. Since bursting behavior continues for several tens of milliseconds, the neuron can not follow the fast change of inputs. In contrast, the performance of the Spiking-AMPA neurons for Cantor coding is high when an input sequence with shorter intervals (about 10-30 msec) is applied.

Two other types of neurons (Spiking-NMDA and Bursting-AMPA) show intermediate characteristics. The Spiking-NMDA neuron is effective for coding when the input time interval is in the range of 100-200 msec. The BurstingAMPA neuron is not as high as others. We confirmed that the cases when $m=3$ and 4 showed similar results.

This different dependency on the input time interval may be advantageous in the encoding events of multiple time scales in the hippocampus.

To investigate how performances of Cantor coding depend on the topology of connections from CA3 to CA1, we used another probability distributions of output patterns $y_{j}^{\sigma}$ in Eq. (1), which are different from the uniform distribution. As an alternative, we set $y_{j}^{\sigma}=1$ with probability $p_{f o}$ and $y_{j}^{\sigma}=0$ otherwise. When $p_{f o}=0.5$, a self-similar distribution of output patterns was observed, and the efficiency of coding was not worth than the case of uniform distribution 
(a) $d=1$, MER $=0$

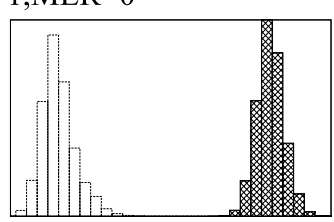

$0 \times 1$ (b) $\mathrm{d}=2, \mathrm{MER}=0.002$

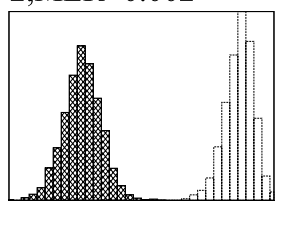

$00 \times 01$

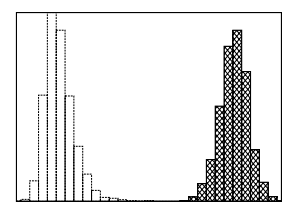

$10 \times 11 \square$

(c) $\mathrm{d}=3, \mathrm{MER}=0.0060$

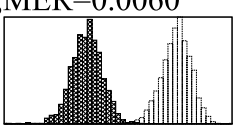

$000 \times 001$

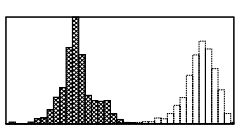

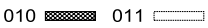

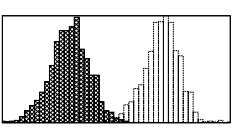

$100 \ldots$

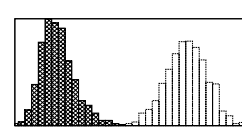

$110 \square$

(d) $\mathrm{d}=4, \mathrm{MER}=0.0315$

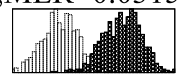

0000

0001

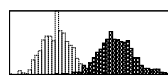

1000 -

e) $d=5, M E R=0.0717$

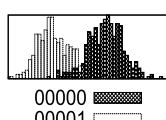

00000
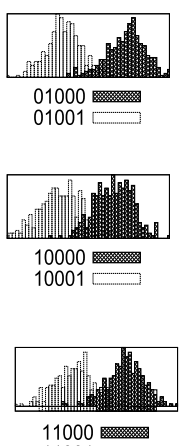

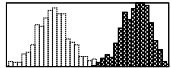

0010 -

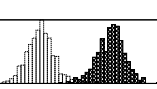

1010 -
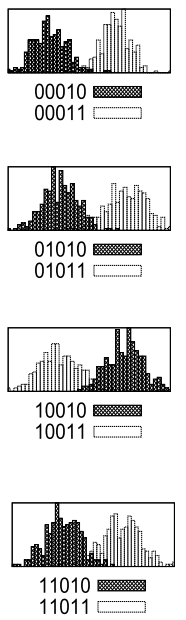

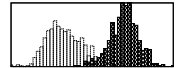

$0100 \div$
$0101 \longleftarrow$

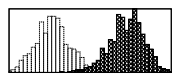

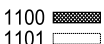
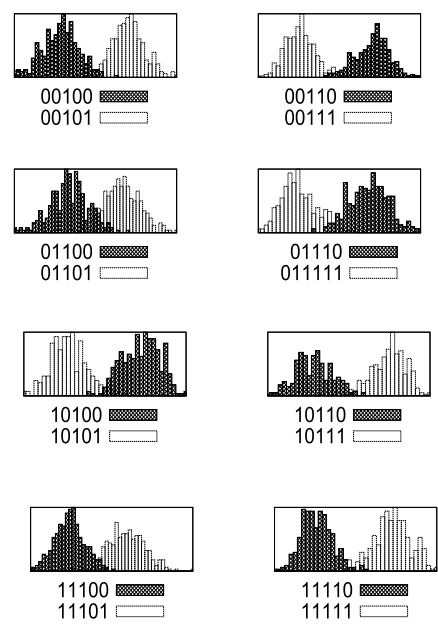

Fig. 8: A representative LDA result. In each box, histograms of projected values $z=A^{T} y$ are shown, where $y$ is the output vector in response to two different input sequences of length $d=1, . ., 5$. Colors represent the two different input sequences. Parameter values are fixed to $m=2, T=100$ and $\tilde{w}=1.6$. 


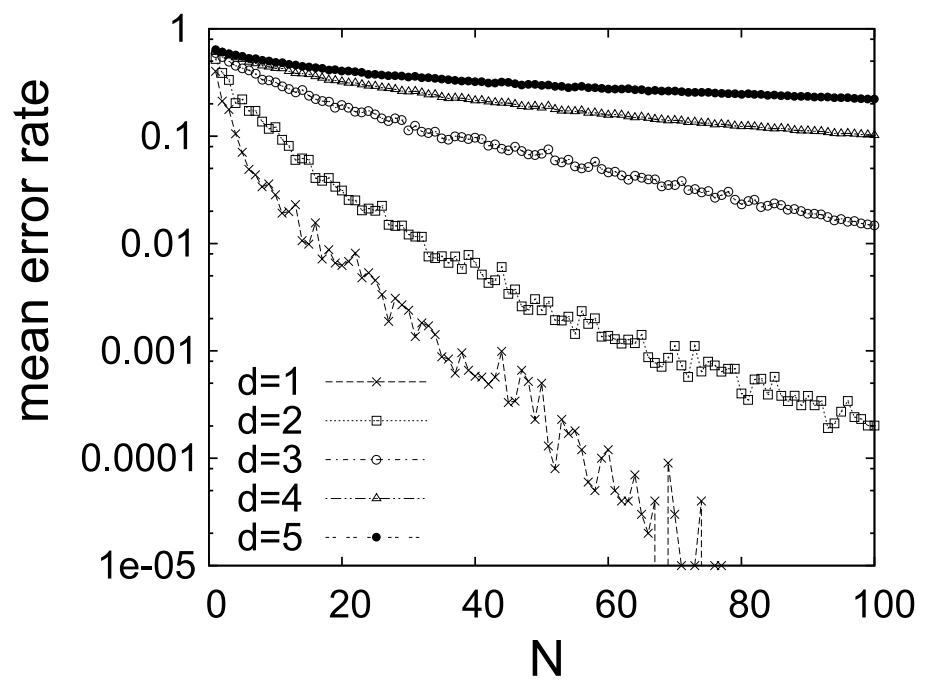

Fig. 9: MER as a function of the number of neurons for $m=3, T=100$, and $\tilde{w}=1.6$. Averages over 10 randomly selected groups are plotted.

(Figures not shown). This means that the assumption of the uniform distribution is not essential to the appearance of Cantor coding. We also investigated the case of sparse coding. When $p_{f o}$ was decreased, the coding performance in the case of spiking outputs becomes worth although the coding performance in the case of membrane potentials is not so bad. Fig. 11 shows error rates as a function of average coupling weights in the case that $y_{j}^{\sigma}$ follows uniform distribution (upper) and the case of sparse coding $p_{f o}=0.2$ (lower). A reason of such a decrease of performance is as follows. When $p_{f_{o}}$ is low, the actual number of neurons participating in the coding by spiking patterns is decreased, and therefore coding performance become worse. In contrast, error rates of decoding from patterns of membrane potentials are not so rapidly decreased because neurons can participate in coding by membrane potentials even though they do not fire.

\section{Summary and Discussion}

In this paper, we clarified the presence of Cantor coding for randomly input spatio-temporal patterns in the CA1 network consisting of neurons described by the two-compartment model. The distributions of spatial firing patterns show fractal-like clustering with hierarchical structure, which reflects the similarity of the input time series. Observing population dynamics of the network, we found that the dynamics can be approximated by a set of contractive affine transformations. Thus, the system can be interpreted as a contractive IFS, in which each transformation is selected by different input patterns. 
The coding performance and its dependence on control parameters were systematically investigated by introducing the error rate of classification as a measure of the coding performance. Using this measure, we found time windows for Cantor coding. In other words, the quality of the coding crucially depends on the time interval of input sequences. In order to efficiently realize the Cantor coding proposed here, the time scale of the input signals must be comparable with the time scale of internal dynamics of the neurons. This relation may determine the critical time interval of the Cantor coding.

Furthermore, using the LDA method, it was clarified that the coding performance was improved by increasing the population of neurons. This result suggests that the Cantor coding in the network is engaged by the population as a whole and not by some specific neurons. Mutual information analysis [42] will be useful to understand the details of such a population coding scheme.

In this paper, we did not treat synaptic learning or the problem of thetaphase precession $[22,32,48]$. The spatio-temporal learning rule in CA1 proposed by Tsukada, Aihara, Saito, \& Kato [41], Tsukada et al. [43] suggests the spatiotemporal representation of input pattern sequences in a single neuron. The relation of our present study to their work is still unclear, and thus remains an issue to be addressed in future study. It is also important to relate the present theory with the theory of the function of phase precession found in the experiments, but this is left for future work as it is beyond the scope of the present study.

\section{Acknowledgments}

This work was supported in part by Grants-in-Aid (Nos. 18019002 and 18047001) for Scientific Research on Priority Areas from the Ministry of Education, Culture, Sports, Science and Technology (MEXT) and the 21st Century COE Program of the Department of Mathematics of Hokkaido University.

\section{Appendix A. The neuron model}

We present the equations for ionic currents in our neuron model Eq. (4). Here, $\tilde{g}_{x}$ is the maximum conductance for the current $I_{x} . V_{\mathrm{Na}}, V_{\mathrm{K}}$ and $V_{\mathrm{Ca}}$ are the reversal potentials for these ionic currents. The leak current is given by

$$
I_{\mathrm{L}}(V)=\tilde{g}_{L}\left(V-V_{L}\right)
$$

The inward sodium current at the soma component is given by

$$
I_{\mathrm{Na}}\left(V_{s}, m, h\right)=\tilde{g}_{\mathrm{Na}} m^{2} h\left(V_{s}-V_{\mathrm{Na}}\right),
$$

where $m$ is the gating variable for fast activation and $h$ is the gating variable for inactivation. The outward delayed-rectifier potassium current is given by

$$
I_{\mathrm{K}-\mathrm{DR}}\left(V_{s}, n\right)=\tilde{g}_{\mathrm{K}-\mathrm{DR}} n\left(V_{s}-V_{\mathrm{K}}\right) .
$$


The inward calcium current is given by

$$
I_{\mathrm{Ca}}\left(V_{d}, s\right)=\tilde{g}_{\mathrm{Ca}} s^{2}\left(V_{d}-V_{\mathrm{Ca}}\right) .
$$

The Ca-activated potassium current is given by

$$
I_{\mathrm{K}-\mathrm{C}}\left(V_{d},\left[\mathrm{Ca}^{2+}\right], c\right)=\tilde{g}_{\mathrm{K}-\mathrm{C}} c \chi\left(\left[\mathrm{Ca}^{2+}\right]\right)\left(V_{d}-V_{\mathrm{K}}\right),
$$

where $\chi\left(\left[\mathrm{Ca}^{2+}\right]\right)=\min \left(\left[\mathrm{Ca}^{2+}\right] / 250,1\right)$. The calcium-dependent potassium current that contributes to the afterhyperpolarization potentials (AHP) is given by

$$
I_{\mathrm{K}-\mathrm{AHP}}\left(V_{d}, q\right)=\tilde{g}_{\mathrm{K}-\mathrm{AHP}} q\left(V_{d}-V_{\mathrm{K}}\right) .
$$

The kinetic equations for each of the gating variables $h, n, s, c$ and $q$ are given by Eq. (5). We list the functions $\alpha$ and $\beta$ below.

$$
\begin{aligned}
& \alpha_{m}\left(V_{s}\right)=\frac{0.32\left(-46.9-V_{s}\right)}{\exp \left(\left(-46.9-V_{s}\right) / 4\right)-1} \\
& \beta_{m}\left(V_{s}\right)=\frac{0.28\left(V_{s}+19.9\right)}{\exp \left(\left(V_{s}+19.9\right) / 5\right)-1} \\
& \alpha_{h}\left(V_{s}\right)=0.128 \exp \left(\left(-43-V_{s}\right) / 18\right) \\
& \beta_{h}\left(V_{s}\right)=\frac{4}{1+\exp \left(\left(-20-V_{s}\right) / 5\right)} \\
& \alpha_{n}\left(V_{s}\right)=\frac{0.016\left(-24.9-V_{s}\right)}{\exp \left(\left(-24.9-V_{s}\right) / 5\right)-1} \\
& \beta_{n}\left(V_{s}\right)=0.25 \exp \left(\left(-40.0-V_{s}\right) / 40.0\right) \\
& \alpha_{s}\left(V_{d}\right)=\frac{1.6}{1+\exp \left(-0.072\left(V_{d}-5\right)\right)} \\
& \beta_{s}\left(V_{d}\right)=\frac{0.02\left(V_{d}+8.9\right)}{\exp \left(\left(V_{d}+8.9\right) / 5\right)-1} \\
& \alpha_{c}\left(V_{d}\right)= \begin{cases}\frac{\exp \left(\left(V_{d}+50\right) / 11\right)-\exp \left(\left(V_{d}+53.5\right) / 27\right)}{18.975} & V_{d}<-10 \\
2 \exp \left(\left(-53.5-V_{d}\right) / 27\right) & V_{d} \geq-10\end{cases} \\
& \beta c\left(V_{d}\right)= \begin{cases}2 \exp \left(\left(-53.5-V_{d}\right) / 27\right)-\alpha_{c}\left(V_{d}\right) & V_{d}<-10 \\
0 & V_{d} \geq-10\end{cases} \\
& \alpha_{q}(C a)=\min (0.00002 C a, 0.01) \\
& \beta_{q}=0.001
\end{aligned}
$$

The parameter values for the bursting type neurons are given below. The reversal potentials are $V_{\mathrm{Na}}=60, V_{\mathrm{K}}=-75, V_{\mathrm{Ca}}=80$, and $V_{L}=-60$. The maximal conductances are $\tilde{g}_{\mathrm{Na}}=30, \tilde{g}_{\mathrm{K}-\mathrm{DR}}=15, \tilde{g}_{\mathrm{Ca}}=10, \tilde{g}_{\mathrm{K}-\mathrm{C}}=15$, and $\tilde{g}_{\mathrm{K}-\mathrm{AHP}}=0.8$. The applied currents are $I_{s}=-0.5$ and $I_{d}=0$. The parameters for coupling between two compartments are $g_{c}=2.1$ and $p=0.5$. The capacitance $C_{m}$ is 3 . The reversal potentials of synaptic currents are $V_{\mathrm{AMPA}}=V_{\mathrm{NMDA}}=0$. For spiking type neurons, we use $\tilde{g}_{\mathrm{Ca}}=2.5$ and $\tilde{g}_{\mathrm{K}-\mathrm{DR}}=25$. Other parameter values are the same as bursting ones. 
The maximal conductances of synaptic currents are $\tilde{g}_{\mathrm{AMPA}}=0.004$ and $\tilde{g}_{\mathrm{NMDA}}=0.01$ for the NMDA type, and $\tilde{g}_{\mathrm{AMPA}}=0.01$ and $\tilde{g}_{\mathrm{NMDA}}=0$ for AMPA type.

\section{Appendix B. Linear Discriminant Analysis}

Assume we are given training data set $\mathcal{Y}=\left\{\mathcal{Y}_{1}, \ldots, \mathcal{Y}_{q}\right\} \subset \mathbb{R}^{N}$, where $\mathcal{Y}_{i}=\left\{y_{1}^{i}, \ldots, y_{l_{i}}^{i}\right\}$ is a set of samples belonging to the class $\omega_{i}(i=1, \ldots, q)$. Linear discriminant analysis (LDA) attempts to find a linear transformation that maximizes the average separation of the projections of points belonging to different classes whilst minimizing the within-class variances of the projections of those points. The transformation is represented by $z=A^{T} y$, where $A$ is an $N \times(q-1)$ matrix. Let the between-class scatter matrix $\Sigma_{B}$ and the within-class matrix $\Sigma_{W}$ be defined as follows:

$$
\begin{aligned}
\Sigma_{B} & =\frac{1}{N} \sum_{i=1}^{q} l_{i}\left(\mu^{i}-\mu\right)\left(\mu^{i}-\mu\right)^{T} \\
\Sigma_{W} & =\frac{1}{N} \sum_{i=1}^{q} \sum_{j=1}^{l_{i}}\left(y_{j}^{i}-\mu^{i}\right)\left(y_{j}^{i}-\mu^{i}\right)^{T},
\end{aligned}
$$

where $\mu^{i}$ is the mean of the samples belonging to class $\omega_{i}$ and $\mu$ is the mean of all the samples. In the lower-dimensional space resulting from the transformation $A$, the two scatter matrices become $\tilde{\Sigma}_{B}=A^{T} \Sigma_{B} A$ and $\tilde{\Sigma}_{W}=A^{T} \Sigma_{W} A$, respectively. The optimal transformation of LDA is given by the matrix $A$ which maximizes

$$
J(A)=\frac{\operatorname{trace}\left(A^{T} \Sigma_{B} A\right)}{\operatorname{trace}\left(A^{T} \Sigma_{W} A\right)} .
$$

Provided that $\Sigma_{W}$ is non-singular, the transformation matrix $A$ consists of the top eigenvectors of $\Sigma_{W}^{-1} \Sigma_{B}$ corresponding to the non-zero eigenvalues, which also satisfies the following equation:

$$
A^{T} \Sigma_{W} A=I,
$$

where $I$ is the identity matrix.

After the transformation matrix is obtained from the training data set, a sample from the test data set $y$ is assigned to the class $\omega_{i}$ whose centroid is closest to the point $z=A^{T} y$ in the low-dimensional space.

[1] Barnsley, M. (1988). Fractals everywhere. Boston: Academic Press.

[2] Buzsáki, G. (2002). Theta oscillations in the hippocampus. Neuron, 33, 325-340.

[3] Connors, B. W. \& Gutnick, M. J. (1990). Intrinsic firing patterns of diverse neocortical neurons. Trends Neurosci., 13, 99-104. 
[4] Csicsvari, J., Jamieson, B., Wise, K. D., \& Buzsáki, G. (2003). Mechanisms of gamma oscillations in the hippocampus of the behaving rat. Neuron, 37, $311-322$.

[5] Eichenbaum, H., Dudchenko, P., Wood, E., Shapiro, M., \& Tanila, H. (1999). The hippocampus, memory, and place cells: Is it spatial memory or a memory space? Neuron, 23, 209-226.

[6] Elman, J. (1990). Finding structure in time. Cogn. Sci., 14, 179-211.

[7] Falconer, K. (1990). Fractal geometry: Mathematical foundations and applications. New York: Wiley.

[8] Fukunaga, K. (1990). Introduction to statistical pattern recognition (2nd ed.). San Diego, CA: Academic Press.

[9] Fukushima, Y., Tsukada, M., Tsuda, I., Yamaguti, Y., \& Kuroda, S. (2007). Spatial clustering property and its self-similarity in membrane potentials of hippocampal CA1 pyramidal neurons for a spatio-temporal input sequence. Cogn. Neurodyn., 1, 305-316.

[10] Hutchinson, J. (1981). Fractals and self-similarity. Indiana Univ. Math. J., 30, $713-747$.

[11] Izhikevich, E. (2003). Simple model of spiking neurons. IEEE Trans. Neural Netw, 14, 1569-1572.

[12] Jarsky, T., Mady, R., Kennedy, B., \& Spruston, N. (2008). Distribution of bursting neurons in the CA1 region and the subiculum of the rat hippocampus. J. Comp. Neurol., 506, 535-547.

[13] Jensen, M., Azouz, R., \& Yaari, Y. (1996). Spike after-depolarization and burst generation in adult rat hippocampal CA1 pyramidal cells. J. Physiol.(Lond.), 492, 199-210.

[14] Jensen, O. \& Lisman, J. E. (1996). Hippocampal CA3 region predicts memory sequences: Accounting for the phase precession of place cells. Learn. Mem., 3, 279-287.

[15] Kesner, R. P., Lee, I., \& Gilbert, P. (2004). A behavioral assessment of hippocampal function based on a subregional analysis. Rev. Neurosci., 15, 333-351.

[16] Leutgeb, S., Leutgeb, J. K., Moser, E. I., \& Moser, M. B. (2006). Fast rate coding in hippocampal CA3 cell ensembles. Hippocampus, 16, 765-774.

[17] Lisman, J. \& Idiart, M. (1995). Storage of 7+/-2 short-term memories in oscillatory subcycles. Science, 267, 1512-1515.

[18] Marr, D. (1971). Simple memory: A theory for archicortex. Philos. Trans. R. Soc. Lond., B, Biol. Sci., 262, 23-81. 
[19] McNaughton, B. L. \& Morris, R. G. M. (1987). Hippocampal synaptic enhancement and information storage within a distributed memory system. Trends Neurosci., 10, 408-415.

[20] Montgomery, S. M. \& Buzsáki, G. (2007). Gamma oscillations dynamically couple hippocampal CA3 and CA1 regions during memory task performance. Proc. Natl. Acad. Sci. U.S.A., 104, 14495-14500.

[21] Nakazawa, K., Quirk, M. C., Chitwood, R. A., Watanabe, M., Yeckel, M. F., Sun, L. D., Kato, A., Carr, C. A., Johnston, D., Wilson, M. A., \& Tonegawa, S. (2002). Requirement for hippocampal CA3 NMDA receptors in associative memory recall. Science, 297, 211-218.

[22] O’Keefe, J. \& Recce, M. (1993). Phase relationship between hippocampal place units and the EEG theta rhythm. Hippocampus, 3, 317-330.

[23] Pinsky, P. F. \& Rinzel, J. (1994). Intrinsic and network rhythmogenesis in a reduced Traub model for CA3 neurons. J. Comput. Neurosci., 1, 39-60.

[24] Pollack, J. (1991). The induction of dynamical recognizers. Mach. Learn., 7, 227-252.

[25] Robinson, C. (1999). Dynamical systems: Stability, symbolic dynamics, and chaos. Boca Raton, FL: CRC Press.

[26] Rössler, O., Knudsen, C., Hudson, J., \& Tsuda, I. (1995). Nowheredifferentiable attractors. Int. J. Intell. Syst., 10, 15-23.

[27] Ryeu, J., Aihara, K., \& Tsuda, I. (2001). Fractal encoding in a chaotic neural network. Phys. Rev. E, 64, 046202.

[28] Scoville, W. B. \& Milner, B. (1957). Loss of recent memory after bilateral hippocampal lesions. J. Neurol. Neurosurg. Psychiatr., 20, 11-21.

[29] Sederberg, P. B., Kahana, M. J., Howard, M. W., Donner, E. J., \& Madsen, J. R. (2003). Theta and gamma oscillations during encoding predict subsequent recall. J. Neurosci., 23, 10809-10814.

[30] Sederberg, P. B., Schulze-Bonhage, A., Madsen, J. R., Bromfield, E. B., McCarthy, D. C., Brandt, A., Tully, M. S., \& Kahana, M. J. (2007). Hippocampal and neocortical gamma oscillations predict memory formation in humans. Cereb. Cortex, 17, 1190-1196.

[31] Siegelmann, H. \& Sontag, E. (1994). Analog computation via neural networks. Theor. Comput. Sci., 131, 331-360.

[32] Skaggs, W., McNaughton, B., Wilson, M., \& Barnes, C. (1996). Theta phase precession in hippocampal neuronal populations and the compression of temporal sequences. Hippocampus, 6, 149-172. 
[33] Squire, L. R., Stark, C. E., \& Clark, R. E. (2004). The medial temporal lobe. Annu. Rev. Neurosci., 27, 279-306.

[34] Tani, J. (1996). Model-based learning for mobile robot navigation from the dynamical systems perspective. IEEE Trans. on Syst. Man Cybern. B Cybern., 26, 421-436.

[35] Treves, A. \& Rolls, E. T. (1994). Computational analysis of the role of the hippocampus in memory. Hippocampus, 4, 374-391.

[36] Tsuda, I. (1992). Dynamic link of memory: Chaotic memory map in nonequilibrium neural networks. Neural Netw., 5, 313-326.

[37] Tsuda, I. (2001). Toward an interpretation of dynamic neural activity in terms of chaotic dynamical systems. Behav. Brain Sci., 24, 793-810.

[38] Tsuda, I., Koerner, E., \& Shimizu, H. (1987). Memory dynamics in asynchronous neural networks. Progr. Theoret. Phys., 78, 51-71.

[39] Tsuda, I. \& Kuroda, S. (2001). Cantor coding in the hippocampus. Japan J. Indust. Appl. Math., 18, 249-258.

[40] Tsuda, I. \& Yamaguchi, A. (1998). Singular-continuous nowheredifferentiable attractors in neural systems. Neural Netw., 11, 927-937.

[41] Tsukada, M., Aihara, T., Saito, H., \& Kato, H. (1996). Hippocampal LTP depends on spatial and temporal correlation of inputs. Neural Netw., 9, $1357-1365$.

[42] Tsukada, M., Ishii, N., \& Sato, R. (1975). Temporal pattern discrimination of impulse sequences in the computer-simulated nerve cells. Biol. Cybern., 17, 19-28.

[43] Tsukada, M., Yamazaki, Y., \& Kojima, H. (2007). Interaction between the spatiotemporal learning rule (STLR) and Hebb type (HEBB) in single pyramidal cells in the hippocampal CA1 Area. Cogn. Neurodyn., 1, 157-167.

[44] Tulving, E. (1972). Episodic and semantic memory. In E. Tulving \& W. Donaldson (Eds.), Organization of memory (pp. 381-403). San Diego, CA: Academic Press.

[45] Whittington, M. A., Traub, R. D., \& Jefferys, J. G. (1995). Synchronized oscillations in interneuron networks driven by metabotropic glutamate receptor activation. Nature, 373, 612-615.

[46] Wills, T. J., Lever, C., Cacucci, F., Burgess, N., \& O’Keefe, J. (2005). Attractor dynamics in the hippocampal representation of the local environment. Science, 308, 873-876. 
[47] Wood, E. R., Dudchenko, P. A., Robitsek, R. J., \& Eichenbaum, H. (2000). Hippocampal neurons encode information about different types of memory episodes occurring in the same location. Neuron, 27, 623-633.

[48] Yamaguchi, Y., Sato, N., Wagatsuma, H., Wu, Z., Molter, C., \& Aota, Y. (2007). A unified view of theta-phase coding in the entorhinal-hippocampal system. Curr. Opin. Neurobiol., 17, 197-204.

[49] Yoshida, M. \& Hayashi, H. (2007). Emergence of sequence sensitivity in a hippocampal CA3-CA1 model. Neural Netw., 20, 653-667.

\section{Vitae}

Yutaka Yamaguti is an assistant professor at the Research Institute for Electronic Science (RIES), Hokkaido University, Japan. He received his B.E. degree from the Department of Engineering, Hokkaido University in 2001, his M.E. degree from the Graduate School of Engineering, Hokkaido University in 2002, and his Ph.D. in Mathematics from the Graduate School of Science, Hokkaido University in 2008. His research interests include computational neuroscience and dynamical systems.

Shigeru Kuroda is a researcher at the Research Institute for Electronic Science (RIES), Hokkaido University, Japan. He received his B.E. degree from the Department of Engineering, Hokkaido University in 1996, his M.E. degree from Graduate School of Science, Hokkaido University in 1998, and his Ph.D. in Mathematics from Graduate School of Science, Hokkaido University in 2009. His research interests include dynamical systems and computational neuroscience.

Yasuhiro Fukushima received the B.S. degree in physics from Waseda University, Tokyo, Japan, in 1995; the M.S. degree in biology from Sophia University, Tokyo, Japan, in 1997, and the PhD degree in biology from Sophia University, in 2003. He is currently a post-doc fellow in the Brain Science Institute, Tamagawa University、Tokyo, Japan. His research interests include physiological function and theoretical description related to learning and memory.

Minoru Tsukada received the B.S.,M.S, and Ph.Dr.dgrees in electrical engineering from Tohoku University, Sendai, Japan in 1965, 1967, and 1971, respectively. MD degree from Yamagata University in 1995. From 1976 to 1985 he was An Associate Professor in the Department of Information Science and Communication Technology, Tamagawa University. He is currently a professor in the Brain Science Research Institute, Tamagawa University. He was the COE Project leader of " Integrative human Science Program " in Japan (2002 - 2007). His research interests are to study the information processing and coding mechanisms of learning and memory from the view point of dynamic systems.

Ichiro Tsuda holds the position of Professor in the Research Institute of Electronic Science (RIES), and the Graduate School of Mathematics, Hokkaido University, as well as Visiting Professor at Department of Mechanical Engineering, Osaka University. He is also the Director of the Research Center for 
Integrative Mathematics, Hokkaido University. He has published widely in the field of chaotic dynamical systems and the brain science. He is also an editor of scientific journals of Neural Networks, Cognitive Neurodynamics, and others, and also an advisory board of Chaos. 


\section{Bursting-NMDA}

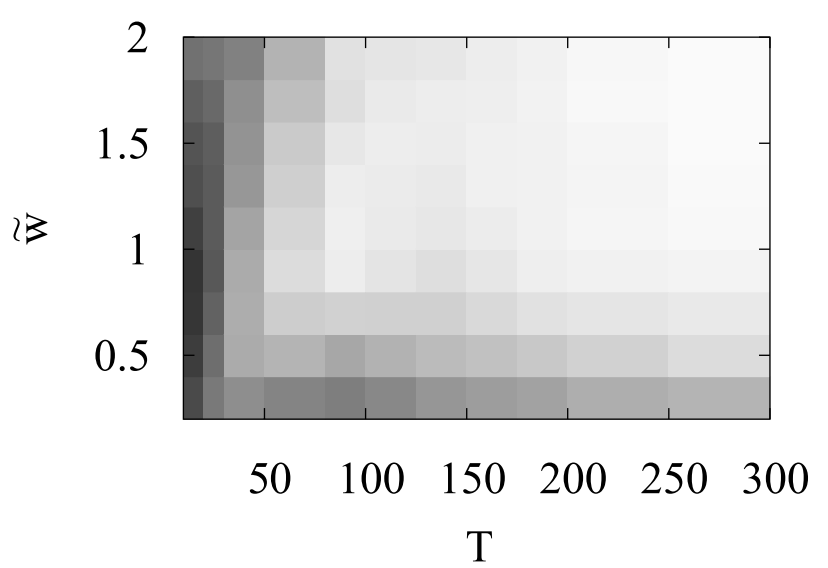

\section{Bursting-AMPA}

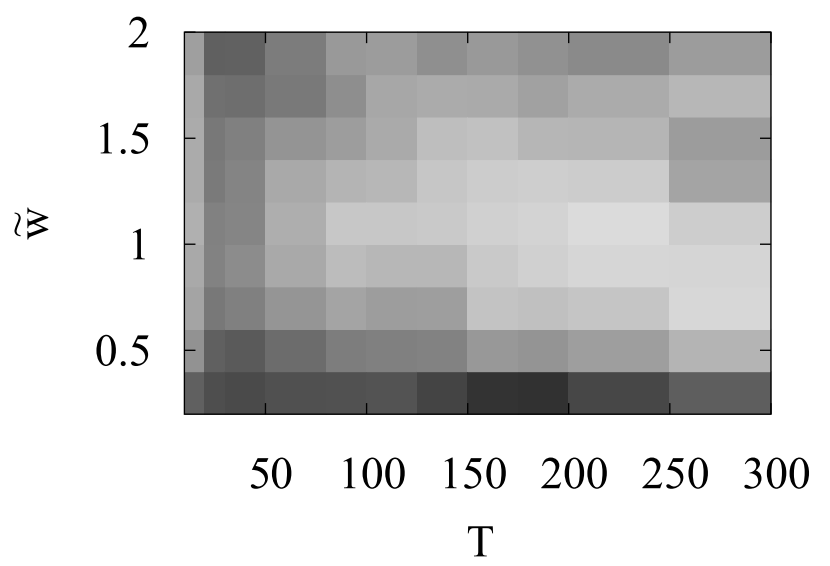

\section{Spiking-NMDA}

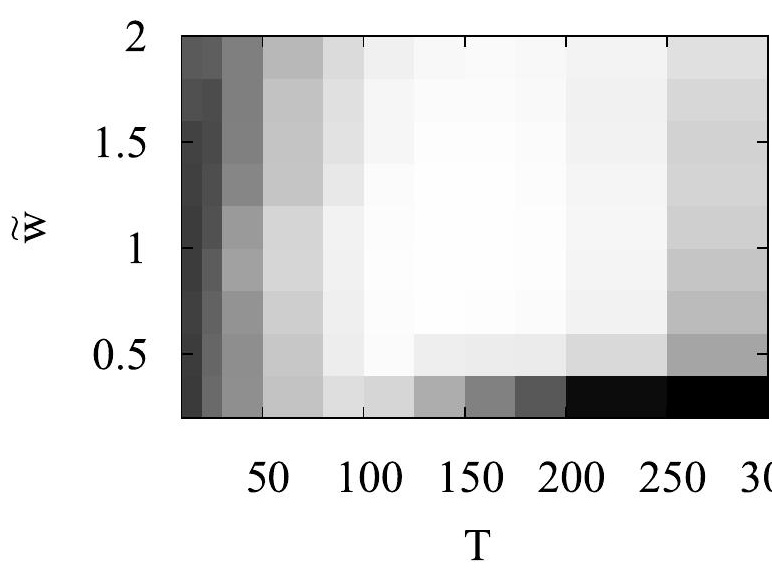

Spiking-AMPA

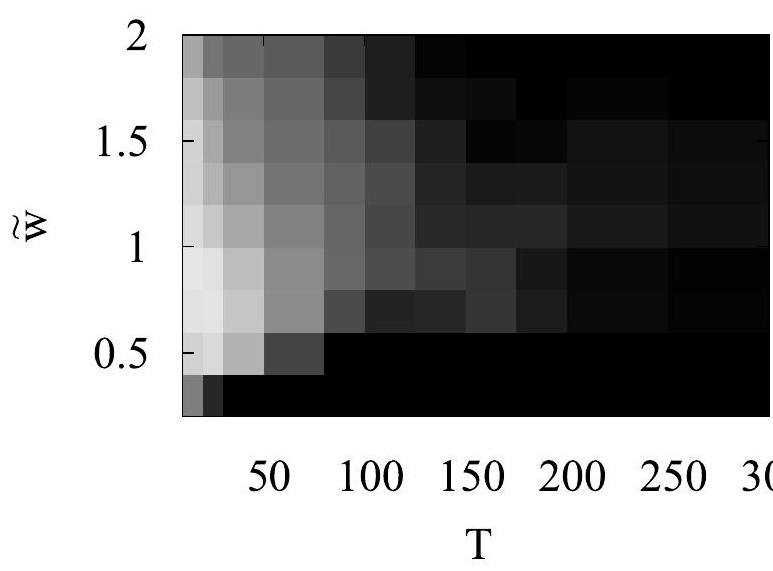

Fig. 10: Dependence of MER on time interval $T$ and input strength $\tilde{w}$. Four networks which consist of neurons with different parameter sets were investigated. Average MERs of five simulations with different connection matrix are shown.

Fig. 11: Error rate in the case that $y_{j}^{\sigma}$ follows uniform distribution (upper) and the case of sparse coding, $p_{f o}=0.2$ (lower). Left figures shows the error rates of decoding from the mean membrane potentials. Right figures shows the error rates of decoding from the output spiking patterns. Black circles denote changes in mean firing rates. 